\title{
Cooperative MIMO-OFDM Communications: Receiver Design for Doppler-Distorted Underwater Acoustic Channels
}

\author{
Kai Tu and Tolga M. Duman \\ School of ECEE \\ Arizona State University \\ Tempe, AZ 85287-5706 \\ Email: \{kai.tu,duman\}@asu.edu
}

\author{
John G. Proakis \\ ECE Department \\ University of California, San Diego, \\ La Jolla, CA 92093, USA \\ Email: proakis@ece.neu.edu
}

\author{
Milica Stojanovic \\ ECE Department \\ Northeastern University \\ 409 Dana, Boston, MA 02115 \\ Email: millitsa@mit.edu
}

\begin{abstract}
Multiple-input multiple-output (MIMO) communications based on orthogonal frequency division multiplexing (OFDM) are considered for improving the performance and bandwidth utilization of underwater acoustic systems in which cooperation is possible between distributed transmitters. The major challenge in such a framework - and the principal difference from the traditional case where multiple transmitters are co-located - is the fact that distributed transmitter-receiver pairs may experience significantly different Doppler distortion (e.g. two vehicles moving in different directions with respect to the receiver). The conventional approach of front-end resampling that corrects for a common Doppler scaling will then fail, rendering a post-FFT signal that is contaminated by transmitter-specific inter-carrier interference. To counteract this problem, we propose a front-end receiver structure that utilizes multiple resampling branches, each followed by FFT demodulation. As a result, a set of sufficient statistics are acquired, which are subsequently processed using custom-designed, linear or nonlinear detection schemes. Numerical results illustrate significant performance improvements as compared to the conventional, single-resampling schemes.
\end{abstract}

\section{INTRODUCTION}

Multiple input multiple output (MIMO) communications based on orthogonal frequency division multiplexing (OFDM) have recently been investigated for underwater acoustic (UWA) systems, as they offer improved utilization of the limited acoustic bandwidth [1], [2]. The extension of these systems to cooperative MIMO-OFDM communications is a promising approach, as user cooperation may bring additional multiplexing/diversity gain that will further boost the overall system performance [3]. However, with the low propagation speed of sound in water (nominally $1500 \mathrm{~m} / \mathrm{s}$ ), the Doppler scaling factors become much greater than those typically observed in terrestrial radio communications, causing significant time variation and intercarrier interference (ICI). When Doppler scaling is similar between various transmitter-receiver pairs, it suffices to resample the received signal once, using an estimate of the (common) Doppler factor. However, this is not the case in a cooperative scenario, where different transmitters may move in different directions with respect to the receiver.

\footnotetext{
${ }^{1}$ This work is funded by the multidisciplinary university research initiative (MURI) grants N00014-07-1-0739/0738, N00014-10-1-0576 and N00014-09-1-0700.
}

In this paper, we consider a $2 \times 2$ cooperative MIMOOFDM system, with two independent data streams sent from two spatially separated transmitters to two co-located receiving elements - extension to larger MIMO configurations is straightforward. The Doppler scaling is assumed to be different for different transmitters, while each transmitter's multipath channel is assumed to have the same Doppler scaling on different propagation paths - extension to path-specific Doppler scaling is also possible.

Prior work on the problem of multiple Doppler scaling includes References [4] and [5], which consider single-input single-output (SISO) systems with path-specific Doppler scaling. Specifically, Ref. [4] points out the importance of acquiring sufficient statistics; however, it focuses on optimizing a single resampling rate, which yields only an approximate solution to the set of sufficient statistics. Our preliminary work [5] proposes a multiple-resampling structure to capture sufficient statistics on a multipath channel with path-specific Doppler scaling.

In contrast to the SISO framework, where the data stream arriving over each propagation path is the same, in the presently considered cooperative MIMO framework, the data stream arriving from each transmitter can be different, implying a receiver structure in which front-end processing occurs in multiple resampling branches, one for each transmitter. As a result, the receiver captures full information available in the MIMO channel, and offers a performance improvement over the conventional receiver that employs a single resampling branch. Specifically, we consider post-FFT processing in the following forms: (1) maximum likelihood (ML) detection, (2) linear detection based on least squares (LS) or minimum mean squared error (MMSE) optimization criterion, and (3) nonlinear detection based on interference cancellation (IC).

The paper is organized as follows. Sec. II contains the $2 \times 2$ MIMO-OFDM system description. In Sec. III, we derive sufficient statistics for detection of multiple data streams, and outline the front-end receiver structure based on efficient FFT implementation. Post-FFT processing is discussed in Sec. IV. Sec. V offers numerical examples that illustrate the system performance, while Sec. VI concludes the paper. 


\section{Cooperative MiMO-OFdM System Model}

The transmitted signal is modeled as

$$
s_{i}(t)=\operatorname{Re}\left\{\sum_{k=0}^{N-1} d_{k}^{(i)} e^{j 2 \pi f_{k} t} R(t)\right\}
$$

where $i=1,2$ is the transmitter index, $d_{k}^{(i)}$ is the data symbol of the $i^{t h}$ transmitter modulated onto the $k^{t h}$ carrier of frequency $f_{k}=f_{0}+k / T$, and $R(t)$ is a rectangular pulse with unity energy and support $\left[-T_{g}, T\right]$. The cyclic prefix (CP) duration $T_{g}$ is assumed to be sufficiently long to prevent interblock interference.

The channel impulse response between the $i^{\text {th }}$ transmitter and the $m^{\text {th }}$ receiver is modeled as

$$
h_{i, m}(t)=\sum_{p=0}^{N_{p}-1} h_{p}^{(i, m)} \delta\left(t+a^{(i)} t-\tau_{p}^{(i, m)}\right)
$$

where $N_{p}$ is the number of paths, $h_{p}^{(i, m)}$ and $\tau_{p}^{(i, m)}$ are respectively the path gain and delay of the $p^{t h}$ path, and $a^{(i)}$ is the Doppler scaling factor associated with the $i^{\text {th }}$ transmitter. Denoting by $v^{(i)}$ the relative speed of the $i$-th transmitter with respect to the centralized receiver, the transmitter-dependent Doppler scaling factor is $a^{(i)}=v^{(i)} / c$, where $c$ is the speed of sound in water. Note that for relative velocities on the order of few meters per second, the values of the Doppler scaling factor can be as high as $10^{-3}$.

Following a similar procedure as in [5], we express the baseband signal of the $m^{t h}$ receiving element as

$$
v_{m}(t)=\sum_{i=1}^{2} \sum_{k=0}^{N-1} d_{k}^{(i)} P_{k}^{(i, m)}(t)+w_{m}(t)
$$

where $w_{m}(t)$ is a circularly-symmetric complex AWGN with power spectral density (PSD) $N_{0}{ }^{1}$ and

$$
\begin{array}{r}
P_{k}^{(i, m)}(t)=\sum_{p=0}^{N_{p}-1} h_{p}^{(i, m)} e^{j 2 \pi f_{k}\left(a^{(i)} t-\tau_{p}^{(i, m)}\right)} \times \\
e^{j 2 \pi k t / T} R\left(t+a^{(i)} t-\tau_{p}^{(i, m)}\right)
\end{array}
$$

\section{Sufficient Statistics}

We consider transmission of a single OFDM block assuming perfect receiver CSI, i.e. the knowledge of Doppler scaling factors as well as the channel path gains and delays. Maximumlikelihood data detection aims to find those sequences $\mathbf{d}^{(1)}=$ $\left[d_{0}^{(1)}, \ldots, d_{N-1}^{(1)}\right]^{T}$ and $\mathbf{d}^{(2)}=\left[d_{0}^{(2)}, \ldots, d_{N-1}^{(2)}\right]^{T}$ which minimize the metric

$$
\begin{aligned}
& \Lambda\left(\mathbf{d}^{(1)}, \mathbf{d}^{(2)}\right)= \\
& \int_{-\infty}^{\infty} \sum_{m=1}^{2}\left|v_{m}(t)-\sum_{i=1}^{2} \sum_{k=0}^{N-1} d_{k}^{(i)} P_{k}^{(i, m)}(t)\right|^{2} d t
\end{aligned}
$$

${ }^{1}$ Noise in an UWA channel is in general colored, but we focus for simplicity on the white noise case as an illustrative example. Extension to a specific noise PSD is straightforward.
The metric (5) implies a set of sufficient statistics which are given by

$y_{k}^{(i, m)}=\int_{-\infty}^{\infty} v_{m}(t) P_{k}^{(i, m)^{*}}(t) d t, \quad k=0,1, \ldots, N-1$

Direct implementation of parallel matched-filter branches for all $N$ carriers is clearly not an option. We thus focus on an alternative interpretation of the expression (6). Namely, we first restrict out attention to the time interval that contains the signal but not its cyclic extension, which effectively yields

$$
y_{k}^{(i, m)} \approx \alpha_{k}^{(i, m)} \tilde{y}_{k}^{(i, m)}
$$

where

$$
\alpha_{k}^{(i, m)}=\sum_{p=0}^{N_{p}-1} \frac{h_{p}^{(i, m)^{*}}}{1+a^{(i)}} e^{j 2 \pi f_{k} \tau_{p}^{(i, m)}}
$$

and

$$
\widetilde{y}_{k}^{(i, m)}=\int_{0}^{\frac{T}{1+a^{(i)}}} v_{m}(t) e^{-j 2 \pi a^{(i)} f_{0} t} e^{-j 2 \pi k\left(1+a^{(i)}\right) t / T} d t
$$

While it may still seem computationally prohibitive to evaluate (9) for all the carriers, this can effectively be performed by a single FFT. To show that this is so, we introduce a change of variable $\xi=\left(1+a^{(i)}\right) t$ in expression (9), which results in

$$
\widetilde{y}_{k}^{(i, m)}=\int_{0}^{T} v_{m}^{(i)}(\xi) e^{-j 2 \pi k \xi / T} d \xi
$$

where

$$
v_{m}^{(i)}(\xi)=v_{m}\left(\frac{\xi}{1+a^{(i)}}\right) e^{-j 2 \pi \frac{a^{(i)}}{1+a^{(i)}} f_{0} \xi}
$$

Hence, the received signal $v_{m}(t)$ can first be resampled and shifted in frequency to obtain the signals $v_{m}^{(1)}(t)$ and $v_{m}^{(2)}(t)$. These signals can now be demodulated according to the expression (10), which, when cast in the discrete-time framework, is nothing but an FFT operation.

Figure 1 shows the block diagram of the receiver front-end. It consists of two (or more) parallel branches, one associated with each transmitter. Each branch requires resampling, frequency shifting, and an FFT operation. The implementation complexity is thus increased only linearly with the number of transmitters, and the processing can be performed in parallel, rending a computationally affordable solution.

\section{DATA DETECTION}

To arrive at the detection algorithms, it is helpful to define an equivalent discrete channel model that relates the acquired statistics (7) to the data symbols. Substituting the relations (3) and (4) into the expression (7), we obtain

$$
y_{k}^{(i, m)}=\sum_{u=1}^{2} \sum_{l=0}^{N-1} H_{k, l}^{(i, u)}(m) d_{l}^{(i)}+w_{k}^{(i, m)}
$$

where

$$
H_{k, l}^{(i, u)}(m)=\int_{0}^{T} P_{l}^{(u, m)}(t) P_{k}^{(i, m)^{*}}(t) d t
$$




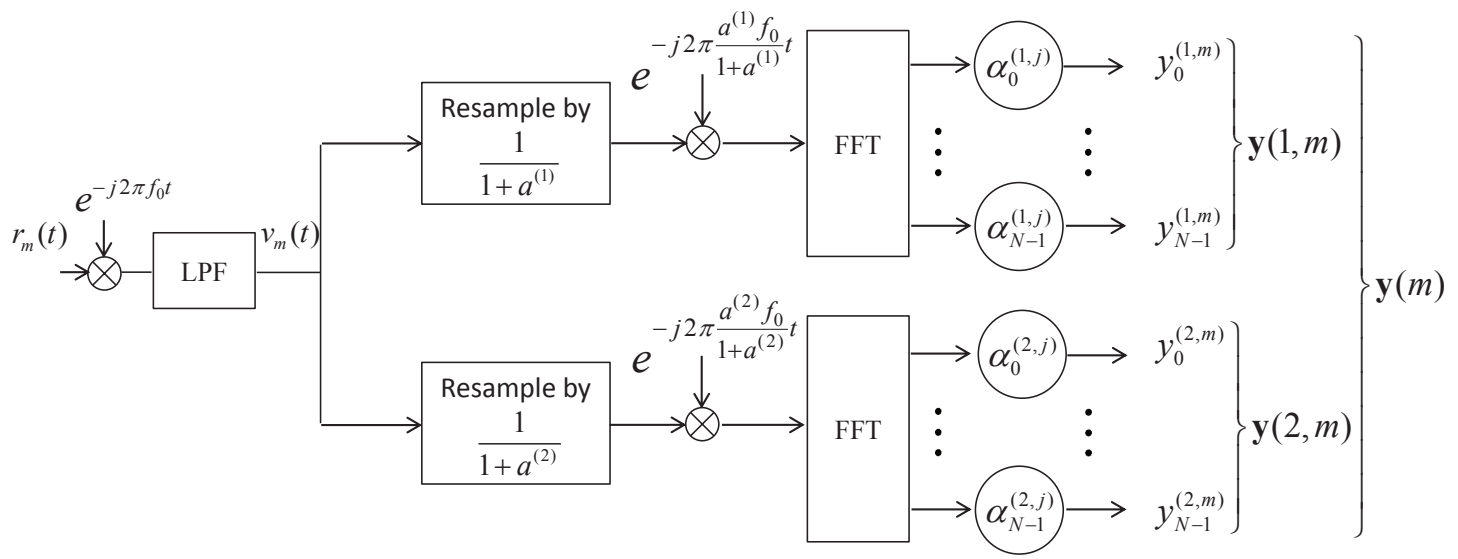

Fig. 1. The multiple-resampling front-end for the $m^{t h}$ receiving element.

and $w_{k}^{(i, m)}$ is additive Gaussian noise with auto-correlation

$$
\Phi_{k, l}^{(i, u)}(m)=E\left[w_{k}^{(i, m)} w_{l}^{(u, m)^{*}}\right]=N_{0} T \cdot H_{k, l}^{(i, u)}(m)
$$

Grouping all the carriers together, the above relationships can also be represented in compact form as

$$
\mathbf{y}(i, m)=\mathbf{H}(i, m) \mathbf{d}+\mathbf{w}(i, m)
$$

where

$$
\begin{gathered}
\mathbf{d}=\left[\mathbf{d}^{(1)^{T}} \mathbf{d}^{(2)^{T}}\right]^{T} \\
\mathbf{H}(i, m)=\left[\mathbf{H}^{(i, 1)}(m) \mathbf{H}^{(i, 2)}(m)\right] \\
{\left[\mathbf{H}^{(i, u)}(m)\right]_{(k, l)}=H_{k, l}^{(i, u)}(m)} \\
\mathbf{y}(i, m)=\left[y_{0}^{(i, m)}, \ldots, y_{N-1}^{(i, m)}\right]^{T} \\
\mathbf{w}(i, m)=\left[w_{0}^{(i, m)}, \ldots, w_{N-1}^{(i, m)}\right]^{T}
\end{gathered}
$$

The vectors $\mathbf{y}(i, m)$ can now be grouped for all the transmitterreceiver pairs to obtain the overall MIMO system model:

$$
\underbrace{\left[\begin{array}{l}
\mathbf{y}(1,1) \\
\mathbf{y}(2,1) \\
\mathbf{y}(1,2) \\
\mathbf{y}(2,2)
\end{array}\right]}_{\mathbf{y}}=\underbrace{\left[\begin{array}{l}
\mathbf{H}(1,1) \\
\mathbf{H}(2,1) \\
\mathbf{H}(1,2) \\
\mathbf{H}(2,2)
\end{array}\right]}_{\mathbf{H}} \mathbf{d}+\underbrace{\left[\begin{array}{l}
\mathbf{w}(1,1) \\
\mathbf{w}(2,1) \\
\mathbf{w}(1,2) \\
\mathbf{w}(2,2)
\end{array}\right]}_{\mathbf{w}}
$$

The aggregate noise vector $\mathbf{w}$ is characterized by the covariance matrix

$$
\boldsymbol{\Phi}=\left[\begin{array}{cc}
\boldsymbol{\Phi}(1) & \mathbf{0} \\
\mathbf{0} & \boldsymbol{\Phi}(2)
\end{array}\right]
$$

with

$$
\boldsymbol{\Phi}(m)=\left[\begin{array}{ll}
\boldsymbol{\Phi}^{(1,1)}(m) & \boldsymbol{\Phi}^{(1,2)}(m) \\
\boldsymbol{\Phi}^{(2,1)}(m) & \mathbf{\Phi}^{(2,2)}(m)
\end{array}\right]
$$

whose entries are defined by (14).

\section{A. Maximum Likelihood Detector and its Approximation}

We pursue ML solution by direct minimization of the original metric in (5), which can be simplified to

$$
\begin{aligned}
\Lambda(\mathbf{d})=C_{0} & -\sum_{m=1}^{2} \sum_{k=0}^{N-1} 2 \operatorname{Re}\left(d_{k}^{(1)^{*}} y_{k}^{(1, m)}+d_{k}^{(2)^{*}} y_{k}^{(2, m)}\right) \\
& +\sum_{m=1}^{2} \sum_{k=0}^{N-1} 2 \operatorname{Re}\left(d_{k}^{(2)^{*}} \sum_{l=0}^{N-1} H_{k, l}^{(2,1)}(m) d_{l}^{(1)}\right)
\end{aligned}
$$

with $C_{0}=\int_{0}^{T} \sum_{m=1}^{2}\left|v_{m}(t)\right|^{2} d t$.

In general, the complexity of ML detection may be prohibitive since the interference ranges over all $N$ carriers. However, the correlation metrics $H_{k, l}^{(2,1)}(m)$ that define coupling of the two transmitted streams in (17) have a certain structure that can be exploited. Namely, if the Doppler scaling factors differ by a small amount, this correlation metric will have only a few significant terms that relate the subcarriers of one transmitter to those of the other. If only the significant terms are kept, an approximate ML detector can be implemented using the Viterbi algorithm. The resulting complexity will be manageable at least for a $2 \times 2$ system. Otherwise, implementation of the Viterbi algorithm becomes infeasible, and suboptimal detectors must be considered.

\section{B. Linear Detectors}

Given (16), we adopt two commonly used linear detectors, the LS detector and the MMSE detector. While conceptually simple, the implementation of these detectors requires some care since the matrices $\mathbf{H}$ and $\boldsymbol{\Phi}$ are both singular. The singularity problem is a direct consequence of the fact that different subsets of the signal vector $\mathbf{y}$ are generated from the same input signal.

The optimal solution can nonetheless be obtained through singular value decomposition (SVD) which removes the redundant coordinates in $\mathbf{y}$ [6]. Although this is an optimal approach, its computational complexity is high. We will thus consider the SVD MMSE detector as a benchmark for optimal linear detection, but will focus instead on two simpler approaches; namely, 
the regularized LS and MMSE detectors. These detectors are defined by ${ }^{2}$

$$
\tilde{\mathbf{d}}_{L S}=\operatorname{Dec}\left(\left(\mathbf{H}^{*} \mathbf{H}+\epsilon \mathbf{I}\right)^{-1} \mathbf{H}^{*} \mathbf{y}\right)
$$

and

$$
\tilde{\mathbf{d}}_{M M S E}=\operatorname{Dec}\left(\mathbf{H}^{*}\left(\mathbf{H H}^{*}+\boldsymbol{\Phi}+\epsilon \mathbf{I}\right)^{-1} \mathbf{y}\right)
$$

where $\operatorname{Dec}(\cdot)$ represents the symbol decision. The regularization factor $\epsilon$ is chosen as a small number with respect to the average of the non-zero eigenvalues of $\mathbf{H H}^{*}$.

\section{Interference Cancelation}

Interference-cancelation (IC) is considered as a means of improving the performance of linear detector. An IC detector forms an estimate of the interference caused by one transmitter to the other, and subtracts this estimate from the desired signal prior to making symbol decisions. The estimation/detection process is performed iteratively, such that the $n^{\text {th }}$ iteration yields an interference estimate

$$
\mathbf{I}_{n}(1, m)=\mathbf{H}^{(1,2)}(m) \tilde{\mathbf{d}}_{I C}^{(2)}(n-1)
$$

which is used to form the symbol decisions as

$$
\tilde{\mathbf{d}}_{I C}^{(1)}(n)=\operatorname{Dec}\left(\sum_{m=1}^{2}\left(\mathbf{Y}(1, m)-\mathbf{I}_{n}(1, m)\right)\right)
$$

The process is analogous for the other transmitter. The IC detector is initialized by symbol decisions obtained using any of the linear detectors discussed previously. Note that after resampling, there is no self-ICI since we only consider Doppler shifts, and the only interference is due to the other transmitter. As numerical examples will illustrate, iterative IC detection offers a significant performance improvement over linear detection while still maintaining a relatively low complexity.

\section{NUMERICAL RESULTS}

To verify the performance of the proposed techniques, a simulation analysis was conducted. Fig. 2 shows the multipath profile of the test channel. The Doppler scaling factors of the two transmitters were set to $-1.0 \times 10^{-3}$, which corresponds to a relative speed of $1.5 \mathrm{~m} / \mathrm{s}$ as the transmitter moves away from the receiver, and $1.2 \times 10^{-3}$, which corresponds to a relative speed of $1.8 \mathrm{~m} / \mathrm{s}$ as the transmitter moves closer to the receiver. Over this channel, two independent 1024carrier OFDM signals were transmitted, occupying the same bandwidth between $12 \mathrm{kHz}$ and $20 \mathrm{kHz}$. The intercarrier spacing is $7.8 \mathrm{~Hz}$, which corresponds an OFDM block duration of $128 \mathrm{~ms}$. A cyclic prefix of length $30 \mathrm{~ms}$ is used, resulting in a complete OFDM block of length $158 \mathrm{~ms}$, which is shaped using a rectangular pulse.

Fig. 3 shows the results of linear detection, focusing on performance comparison between the multiple-resampling and the single-resampling front-end. Single-resampling includes resampling according to the Doppler scaling factor of the first

\footnotetext{
${ }^{2}$ Symbol “*” denotes Hermitian transpose.
}
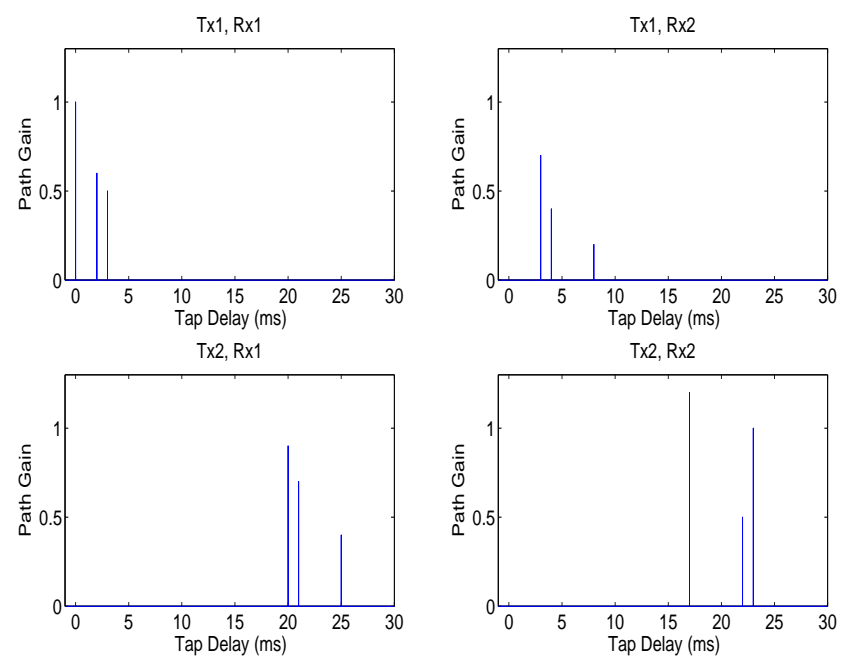

Fig. 2. Multipath profile of the test channel.

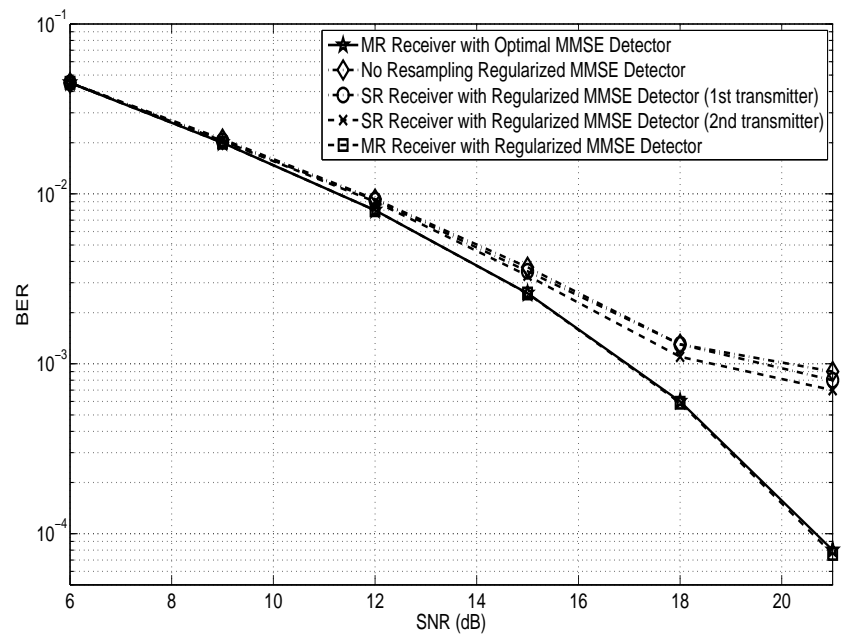

Fig. 3. Performance of linear detection with multiple-resampling (MR) and single-resampling (SR) front-end.

transmitter and that of the second transmitter. Included also is the case with no resampling. Note that with the transmitters' Doppler scaling factors close in magnitude and opposite in sign, the receiver with no resampling can be interpreted as a special case of the single-resampling receiver [4], whose resampling rate is roughly the average of the two. The results of Fig. 3 are obtained using the regularized MMSE detector with $\epsilon=0.005$ (the average of the non-zero eigenvalues of $\mathbf{H H}^{*}$ is 0.086). Included also are the results for the optimal, SVD MMSE detector. The regularized MMSE detector with multiple resampling obviously performs very close to the optimum. More importantly, it offers a substantial performance gain over the single-resampling detectors.

Fig. 4 shows the performance of various detectors proposed for the multiple-resampling receiver. Included are the regularized MMSE detector, the genie-aided IC detector, in which 


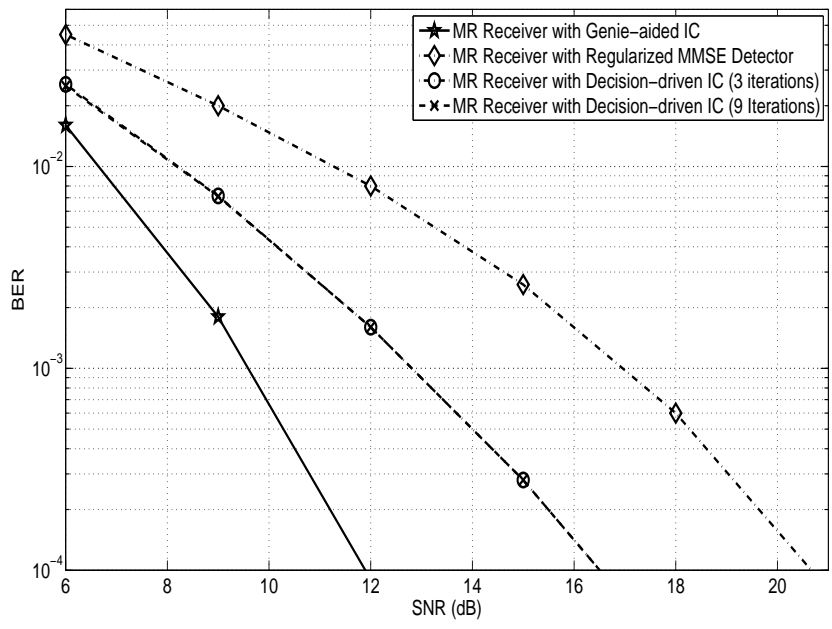

Fig. 4. Performance of multiple resampling with linear and nonlinear detection schemes.

the interference estimate is made using known symbols from the interfering transmitter, and the decision-driven IC detector. The latter is initialized with regularized MMSE decisions, and employs 3 or 9 iterations. Clearly, the IC detector provides performance that is closer to the genie-aided IC bound, outperforming the regularized MMSE detector by $3 \mathrm{~dB}$ or more. The IC detector takes only three iterations to converge, thus offering a good compromise between performance and complexity. The complexity of ML detection, even in the approximate form, is prohibitive for this test channel (at least 4096 states are required in the Viterbi algorithm).

\section{CONCLUSIONS}

Motivated by the problem of disparate transmitter-specific Doppler distortion in a cooperative mobile acoustic system, we developed an optimum receiver front-end that secures acquisition of sufficient statistics through multiple-resampling branches. Unlike the existing designs that resample the received signal once, the proposed structure capitalizes on the knowledge of transmitter-specific Doppler scales to improve the system performance. Coupled with custom-designed linear or nonlinear post-processing, it is shown to offer a significant performance gain over conventional detection, thus justifying the (moderate) complexity increase. Future research will address cooperative diversity with distributed space-time or space-frequency coding in the presence of transmitter-specific Doppler scaling, as well as Doppler estimation and its practical demonstration.

\section{REFERENCES}

[1] B. Li, J. Huang, S. Zhou, K. Ball, M. Stojanovic, L. Freitag, and P. Willett, "MIMO-OFDM for High Rate Underwater Acoustic Communications,", IEEE Journal of Oceanic Engineering, vol. 34, no. 4, pp. 634-645, Oct. 2009.

[2] M. Stojanovic, "MIMO OFDM Over Underwater Acoustic Channels,", in Proc. 43rd Asilomar Conference on Signals, Systems and Computers, Pacific Grove, California, Nov. 2009, pp. 605-609.
[3] M. Vayapeyam, S. Vedentam, U. Mitra, J. Preisig, and M.Stojanovic, "Distributed Space-Time Cooperative Schemes for Underwater Acoustic Communications,", IEEE Journal of Oceanic Engineering, vol. 33, no. 4, pp. 489-501, 2008.

[4] S. Yerramalli and U. Mitra, "On Optimal Resampling for OFDM Signaling in Doubly-Selective Underwater Acoustic Channels," in Proc. IEEE Oceans 2008, Quebec City, Canada, Sep. 2008, pp. 1-6.

[5] K. Tu, D. Fertonani, and T. M. Duman, "OFDM Receiver for Underwater Acoustic Channels with Non-Uniform Doppler Arrivals," in Proceedings of the 10th European Conference on Underwater Acoustics, Istanbul, Turkey, Jul. 2010, pp. 973-978.

[6] G. H. Golub and C. F. V. Loan, Matrix Computations, 3rd ed. Baltimore: Johns Hopkins University Press, 1999. 